\title{
Gemcitabine reactivates epigenetically silenced genes and functions as a DNA methyltransferase inhibitor
}

\author{
STEVEN G. GRAY ${ }^{1}$, ANNE-MARIE BAIRD ${ }^{1}$, FARDOD O'KELLY ${ }^{2}$, GEORGIOS NIKOLAIDIS ${ }^{3}$, \\ MALIN ALMGREN $^{4}$, ARMELLE MEUNIER ${ }^{2}$, EILIS DOCKRY ${ }^{1}$, DONAL HOLLYWOOD ${ }^{2}$, \\ TOMAS J. EKSTRÖM ${ }^{4}$, ANTOINETTE S. PERRY ${ }^{2}$ and KENNETH J. O'BYRNE ${ }^{1,5}$
}

\author{
${ }^{1}$ Thoracic Oncology Research Group, Institute of Molecular Medicine, Trinity College Dublin, Dublin 2; \\ ${ }^{2}$ Prostate Molecular Oncology, Trinity Centre for Health Sciences, St. James's Hospital, Dublin 8, Republic of Ireland; \\ ${ }^{3}$ Roy Castle Lung Cancer Research Program, Department of Molecular and Clinical Cancer Medicine, \\ University of Liverpool Cancer Research Centre, Liverpool L3 9TA, UK; ${ }^{4}$ Department of Clinical Neuroscience, \\ Karolinska Institute, Center for Molecular Medicine, SE-17176 Stockholm, Sweden; \\ ${ }^{5}$ HOPE Directorate, St. James's Hospital, Dublin 8, Republic of Ireland
}

Received July 4, 2012; Accepted August 6, 2012

DOI: $10.3892 /$ ijmm.2012.1138

\begin{abstract}
Gemcitabine is indicated in combination with cisplatin as first-line therapy for solid tumours including non-small cell lung cancer (NSCLC), bladder cancer and mesothelioma. Gemcitabine is an analogue of pyrimidine cytosine and functions as an anti-metabolite. Structurally, however, gemcitabine has similarities to 5-aza-2-deoxycytidine (decitabine/ Dacogen ${ }^{\circledR}$ ), a DNA methyltransferase inhibitor (DNMTi). NSCLC, mesothelioma and prostate cancer cell lines were treated with decitabine and gemcitabine. Reactivation of epigenetically silenced genes was examined by RT-PCR/ qPCR. DNA methyltransferase activity in nuclear extracts and recombinant proteins was measured using a DNA methyltransferase assay, and alterations in DNA methylation status were examined using methylation-specific PCR (MS-PCR) and pyrosequencing. We observe a reactivation of several epigenetically silenced genes including GSTP1, IGFBP3 and RASSF1A. Gemcitabine functionally inhibited DNA methyltransferase activity in both nuclear extracts and recombinant proteins. Gemcitabine dramatically destabilised DNMT1 protein. However, DNA CpG methylation was for the most part unaffected by gemcitabine. In conclusion, gemcitabine both inhibits and destabilises DNA methyltransferases and reactivates epigenetically silenced genes having activity equivalent to decitabine at concentrations significantly lower than those achieved in the treatment of patients with solid tumours. This property may contribute to the anticancer activity of gemcitabine.
\end{abstract}

Correspondence to: Dr Steven G. Gray, Thoracic Oncology Research Group, Institute of Molecular Medicine, Trinity College Dublin 2, Republic of Ireland

E-mail: sgray@stjames.ie

Key words: cancer, gemcitabine, DNA methyltransferase, epigenetics, DNMT inhibitor

\section{Introduction}

Gemcitabine is a well-established anticancer agent and has been FDA approved either as a single agent for the treatment of pancreatic cancer (1) or in combination with another agent (carboplatin, cisplatin or paclitaxel) for the treatment of ovarian (2) and metastatic breast cancer (3). Cisplatin is indicated as first-line therapy for inoperable, locally advanced (stage IIIA or IIIB) or metastatic (stage IV) non-small cell lung cancer (NSCLC) $(4,5)$, and has also been studied in other solid tumours such as malignant pleural mesothelioma (MPM) $(6,7)$ and prostate cancer $(8,9)$.

Gemcitabine is considered to act as a pyrimidine-type anti-metabolite. The agent is a prodrug and requires intracellular conversion to two active metabolites, gemcitabine diphosphate and gemcitabine triphosphate, which can function in two ways: by binding to and irreversibly inhibiting ribonucleotide reductase (RNR) and by replacing one of the building blocks of nucleic acids, in this case cytosine, during DNA replication (10).

Aberrant epigenetic regulation of gene expression is a frequent event in cancer (11). Several of the proteins which regulate histone post-translational modifications have now been shown to play a role in resistance to various cancer therapies including cisplatin (12), gefitinib (13), etoposide (14) and tamoxifen (15).

DNA CpG methylation is another epigenetic modification that is linked to loss of gene expression in cancer (16). Inhibitors targeting DNA methyltransferases have been developed. Of these 5-azacytidine $\left(\right.$ Vidaza $^{\circledR}$ ) and 5-aza2-deoxycytidine (decitabine/Dacogen ${ }^{\circledR}$ ) have gained FDA approval for the treatment of myelodysplastic syndrome subtypes $(17,18)$.

Notably, the chemotherapy agent doxorubicin has been shown to inhibit the DNA methyltransferase DNMT1 (19) and indicates that other chemotherapy drugs may potentially inhibit the epigenetic enzymatic machinery. Since decitabine 
and gemcitabine are cytosine analogues, and share similar structural features (Fig. 1), we reasoned that gemcitabine may also be able to act as a DNA methyltransferase inhibitor (DNMTi).

\section{Materials and methods}

Cell lines. The A549 (adenocarcinoma), SKMES1 (squamous cell carcinoma), H-28 (MPM), 22Rv1 (prostate), LNCaP (prostate) and Du145 (prostate) cell lines were purchased from the ATCC (LGC Promochem). Cells were cultured in appropriate media and maintained at $37^{\circ} \mathrm{C}$ in a humidified atmosphere containing $5 \% \mathrm{CO}_{2}$.

Cell line treatments. Decitabine was purchased from Merck and dissolved in methanol. Cell cultures were treated for $48 \mathrm{~h}$ at a final concentration of $5 \mu \mathrm{M}$, with the media and drug replaced every $24 \mathrm{~h}$ as previously described (20).

Gemcitabine was supplied by Eli Lilly and dissolved in phosphate-buffered saline (PBS) at a final concentration of $38 \mathrm{mg} / \mathrm{ml}$ (120.14 mM). Cell cultures were treated for $48 \mathrm{~h}$ at final concentrations of $0.2,0.5$ or $1 \mu \mathrm{M}$ with the media and drug replaced every $24 \mathrm{~h}$. We chose to use this concentration based on literature searches in Pubmed.

RNA isolation and RT-PCR amplification. Total RNA was extracted using TRI Reagent ${ }^{\circledR}$ (MRCgene) according to the manufacturer's instructions. One microgram of total RNA was used to generate cDNA using M-MLV-reverse transcriptase (Promega) according to the manufacturer's instructions.

Expression of VEGFR1, VEGFR2, sFRP4, RASSF1A and $\beta$-actin was examined by RT-PCR, using primers and PCR conditions outlined in Table I. Each PCR was carried out for 35 cycles. Ten microlitres of the experimental RT-PCR product and $2 \mu \mathrm{l}$ of the $\beta$-actin RT-PCR product were loaded onto a $1 \%$ agarose gel.

Quantitative PCR. RNA was isolated from the cell lines using a miRVana miRNA isolation kit (Applied Biosystems, UK) according to the manufacturer's guidelines. Total RNA $(1 \mu \mathrm{g})$ was reverse transcribed using the high capacity cDNA Archive kit. Expression of GSTP1 (TaqMan ${ }^{\circledR}$ gene expression assay ID: Hs00168310_m1) and IGFBP3 (TaqMan gene expression assay ID: Hs00181211_m1) was quantified in the cell lines by qRT-PCR. Human phosphoglycerate kinase 1 (PGK1) was used as an endogenous control (TaqMan gene expression assay ID: Hs99999906_m1). TaqMan PCR reactions were performed in triplicate on an ABI Prism 7900 sequence detection system. Gene expression was calculated relative to the untreated cell lines, using SDS RQ Manager 1.2 software, which automatically determines relative quantities (RQ), by applying the arithmetic formula $2^{-\Delta \Delta C T}$. All equipment and reagents were supplied by Applied Biosystems, Foster City, CA.

DNA methyltransferase assay. Analysis of DNA methyltransferase activity was carried out using the EpiQuik ${ }^{\mathrm{TM}}$ DNA methyltransferase activity/inhibition assay kit (Epigentek) using both recombinant DNMTs (Epigentek) and nuclear extracts isolated with the EpiQuik Nuclear Extraction kit II
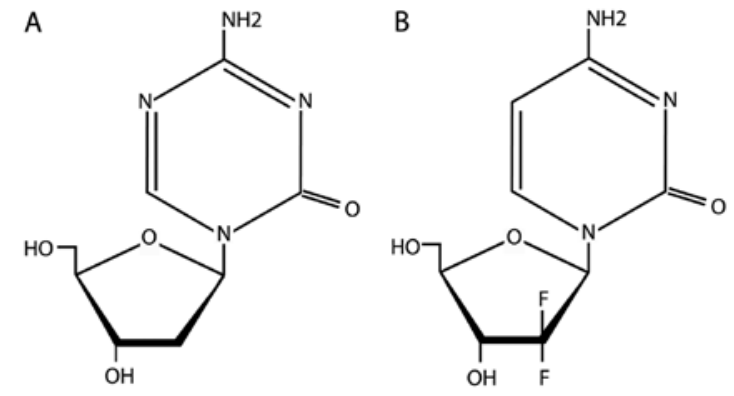

Figure 1. Chemical structures of (A) 5-aza-2-deoxycytidine (decitabine) and (B) gemcitabine.

(Epigentek) according to the manufacturer's instructions. Following consultation with the manufacturers, the recombinant proteins were incubated with the various concentrations of decitabine or gemcitabine for $90 \mathrm{~min}$ at room temperature prior to conducting the methyltransferase assay.

Genomic DNA isolation, bisulfite conversion and MS-PCR analysis. Genomic DNA was isolated from the cell lines using a solution containing $0.5 \%$ SDS and $100 \mu \mathrm{g} / \mathrm{ml}$ Proteinase K (21).

Genomic DNA from the cell lines (500 ng) was bisulfite modified using the EpiTect Bisulfite kit (Qiagen, UK), following the manufacturer's guidelines. EpiTect methylated DNA and unmethylated DNA were used as controls.

Promoter hypermethylation of the GSTP1 promoter was analysed by methylation-specific PCR (MS-PCR) in the LNCaP and 22Rvl cell lines using the GoTaq HotStart enzyme (Promega). PCR primer sets complementary to both modified, methylated DNA (M) and modified, unmethylated DNA (U) were designed for GSTP1: GSTP1 MF2, 5'-TTCGGGGGTGTA GCGGTCGTC-3'; GSTP1 MR1, 5'-CCAACGAAAACCTCGC GACCTCCG-3' (expected product, 145 bp); GSTP1 UF2, 5'-GATGTTTGGGGTGTAGTGGTTGTT-3'; GSTP1 UR1, 5'AAACTCCAACAAAAACCTCACAACCTCCA-3' (expected product, $154 \mathrm{bp})$.

Bisulfite pyrosequencing. Pyrosequencing methylation analysis (PMA) of the LINE-1.2 element, the RASSF1A promoter and the VEGFR gene promoters, was performed as previously described (22-24). Genomic DNA (500-1,000 ng) was bisulfite treated, using the EpiTect Bisulfite kit or the EZ DNA Methylation $^{\text {TM }}$ kit (ZymoResearch). The PyroMarkAssay Design software was used to design the primers for amplification and sequencing to cover a number of $\mathrm{CpG}$ sites as shown in Table II. PCR amplification products were cleaned and subjected to pyrosequencing on either a PyroMark Q96 ID pyrosequencer using PyroMark Gold Q96 SQA reagents (all were from Qiagen), or on a Q24 pyrosequencer according to the manufacturer's protocol (Biotage). The methylation of $\mathrm{C}$ in each analysed $\mathrm{CpG}$ site was quantified from 0 to $100 \%$, using the PyroMark software (Qiagen).

Western blot analysis. Protein lysates were extracted from cell cultures using RIPA buffer [50 mM Tris $\mathrm{HCl}, \mathrm{pH} 7.4,150 \mathrm{mM}$ $\mathrm{NaCl}, 1 \mathrm{mM}$ EDTA, $1 \%$ (v/v) Triton X-100, 0.1\% (w/v) SDS], supplemented with $10 \mu \mathrm{l}$ phenylmethylsulfonyl fluoride 
Table I. Primers and annealing temperatures for RT-PCR.

\begin{tabular}{llc}
\hline Primer Set & \multicolumn{1}{c}{ Sequence } & Annealing Temp $\left({ }^{\circ} \mathrm{C}\right) / \mathrm{PCR}$ cycles \\
\hline VEGFR1 & F: 5'-CAAGTGGCCAGAGGCATGGAGTT-3' & $56^{\circ} \mathrm{C} / 35 \mathrm{cycles}$ \\
& R:5'-GATGTAGTCTTTACCATCCTGTTG-3' & \\
VEGFR2 & F: 5'-GAGGGCCTCTCATGGTGATTGT-3' & $56^{\circ} \mathrm{C} / 35 \mathrm{cycles}$ \\
RFRP4 & R: 5'-TGCCAGCAGTCCAGCATGGTCTG-3' & $56^{\circ} \mathrm{C} / 35 \mathrm{cycles}$ \\
& F: 5'-TCTATGACCGTGGCGTGTGC-3' & $56^{\circ} \mathrm{C} / 35 \mathrm{cycles}$ \\
RASSF1A & R: 5'-ACCGATCGGGGCTTAGGCGTTTAC-3' & \\
& F: 5'-CAGATTGCAAGTTCACCTGCCACTA-3' & $56^{\circ} \mathrm{C} / 35 \mathrm{cycles}$ \\
\end{tabular}

F, forward; R, reverse.

Table II. Primers for bisulfite pyrosequencing.

LINE1.2 (6 CpGs)
LINE-1 fwd: 5'-BIO-TAGGGAGTGTTAGATAGT GG-3',
LINE-1 rev: 5'-AACTCCCTAACCCCTTAC-3',
LINE-1 seq: 5'-CAAATAAAACAATACCTC-3'.
RASSF1A (9 CpGs)
RASSF1 methF: 5'-AGTATAGTAAAGTTGGTTTTTAGAAA-3'
RASSF1 methR: 5'-CCCTTCCTTCCCTCCTT-3'
RASSF1 methPSEQ: 5'-AAGTTGGTTTTTAGAAATA-3'
VEGFR1 (15 CpGs)
VEGFR1 PyroF: 5'-AGGAGGGGTAAGGGTAAGAG-3'
VEGFR1 PyroR: 5'-TCCCCACCTACCCTCTTCTT-3'
VEGFR1 PyroSEQ: 5'-GGGAGAGGAGTAAAGATTTTGAATT-3'

(87 $\mathrm{mg} / \mathrm{ml}$ in $96 \% \mathrm{EtOH}$ ) and $100 \mu \mathrm{l}$ protease inhibitor cocktail ( $2 \mathrm{mM}$ AEBSF, $1 \mathrm{mM}$ EDTA, $130 \mu \mathrm{M}$ bestatin, $14 \mu \mathrm{M}$ E-64, $1 \mu \mathrm{M}$ leupepin, $0.3 \mu \mathrm{M}$ aprotinin). Lysates were separated by SDS/PAGE and subsequently transferred onto pre-activated polyvinylidene fluoride nitrocellulose membranes (PVDF). Membranes were blocked for $1 \mathrm{~h}$ at room temperature (RT) in TBST $[10 \mathrm{mM}$ Tris- $\mathrm{HCl}(\mathrm{pH} 7.5)$, $10 \mathrm{mM} \mathrm{NaCl}, 0.1 \%$ (v/v) Tween 20] containing 5\% nonfat dry milk powder. Membranes were immunoblotted overnight at $4^{\circ} \mathrm{C}$ in TBST with $5 \%$ nonfat dry milk powder with DNMT1 (supplier) with HDAC1 (Cell Signalling Technologies) used as a loading control as appropriate. All secondary antibodies were HRP-labelled and bound antibody complexes were detected using the Supersignal West Pico Chemiluminescent kit (Pierce, Rockford, IL, USA).

\section{Results}

Gemcitabine reactives $m R N A$ expression of epigenetically silenced genes. A549 (NSCLC), H28 (MPM), LNCaP (prostate) and 22Rv1 (prostate) cells were treated with either decitabine or gemcitabine and the effects of these drugs on gene expression were examined. In A549 cells decitabine and gemcitabine induced both VEGFR1 and VEGFR2 (Fig. 2A). In the $\mathrm{H} 28$ cell line a gene previously shown to be epigenetically silenced in this cell line by DNA CpG methylation $(25,26)$, sFRP4, was reactivated by both drugs (Fig. 2B). Both GSTP1 and IGFBP3 have been shown to be epigenetically silenced by DNA CpG methylation in prostate cancer by us and others (27-29). Using quantitative PCR we measured the effect of decitabine and gemcitabine on these genes in two prostate cancer cell lines (LNCaP and 22Rv1). Both drugs were shown to significantly induce expression of GSTP1 (Fig. 2C) and IGFBP3 (Fig. 2D).

As all these genes were examined separately in different cell lines we reviewed the literature to find whether there was a common gene frequently silenced by DNA CpG methylation in lung, MPM and prostate cell lines. From this analysis we chose the gene RASSF1A which was shown to be silenced by methylation in several of our cell lines (30-32). Gemcitabine was able to reactivate/upregulate RASSF1A in 3 out of the 4 cell lines tested whereas decitabine reactived RASSF1A in 4 out of the 4 cell lines at all concentrations tested (Fig. 3).

Decitabine functions in vitro and in vivo to inhibit DNA methyltransferases. A DNA methyltransferase activity/inhibition assay was used to measure the effects of gemcitabine on DNMT enzymatic activity, with decitabine used as a positive inhibitor control. Using recombinant DNMT protein mixture we demonstrated that both decitabine and gemcitabine inhibited DNMT activity (Fig. 4A and B). Furthermore, DNMT activity was also inhibited in nuclear extracts from cells exposed to either decitabine or gemcitabine (Fig. 4C and D), indicating that gemcitabine inhibits DNA methyltransferase activity.

Gemcitabine affects DNMT1 protein stability. Decitabine has been shown to selectively degrade DNMT1 protein levels by a proteasomal-based pathway (33). To examine whether gemcitabine also affects the levels of DNMT1 protein, western blot analyses were carried out on extracts from 


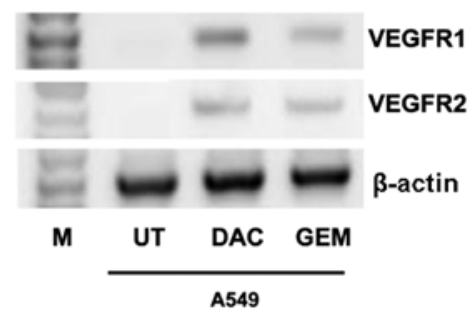

B

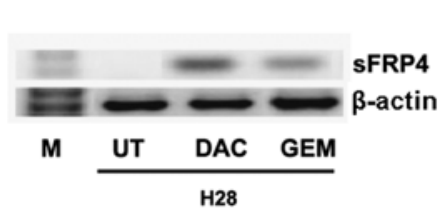

C

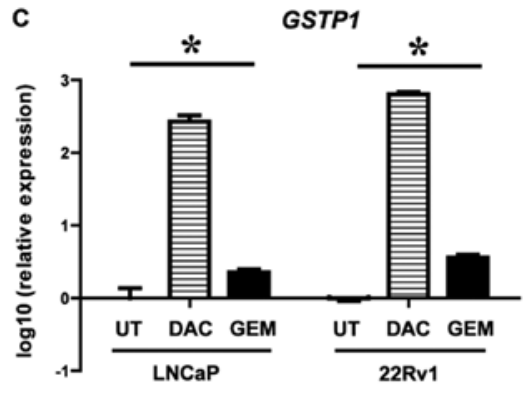

D

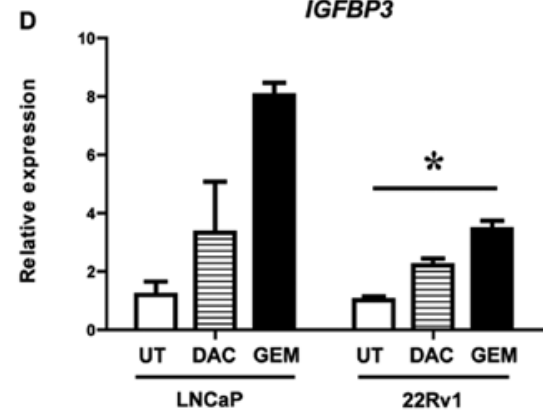

Figure 2. Reactivation of silenced genes by gemcitabine in cancer cell lines. A549 (NSCLC), H28 (MPM), 22Rv1 and LNCaP cells were treated with either decitabine (final concentration $5 \mu \mathrm{M}$ ) or gemcitabine (final concentration $1 \mu \mathrm{M}$ ) for $48 \mathrm{~h}$ with the media and drugs being replaced every $24 \mathrm{~h}$. Reactivation of gene expression in the cell lines was monitored using either RT-PCR or qPCR. Results are provided for (A) VEGFR1 and VEGFR2 in A549 cells, (B) sFRP4 in $\mathrm{H} 28$ cells, (C) GSTP1 $(\mathrm{p}<0.05)$ and (D) IGFBP3 $(\mathrm{P}<0.05)$. The results clearly demonstrated that these genes were reactivated in response to treatment with either drug. Statistical significance was determined using the non-parametric Kruskal-Wallis test.

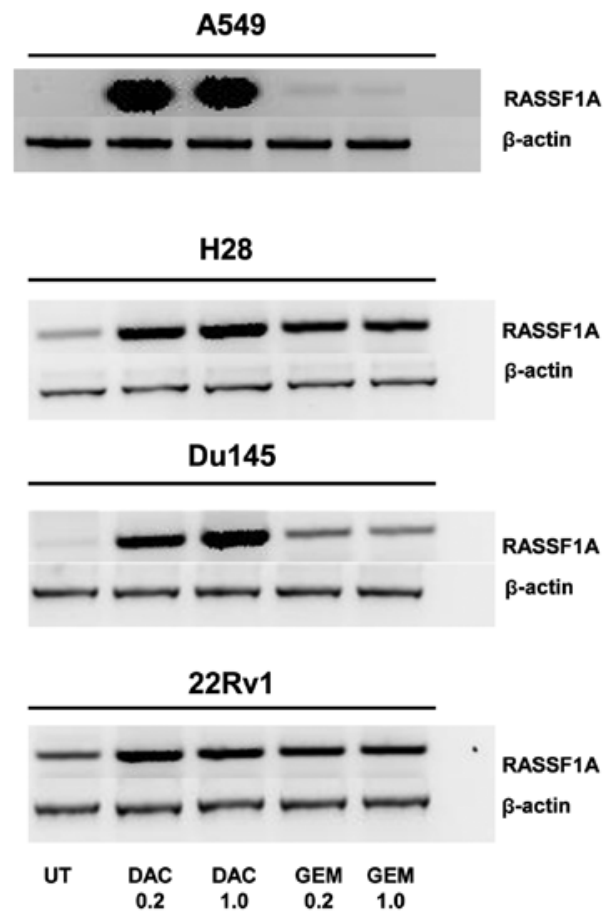

Figure 3. Reactivation of a commonly silenced gene by gemcitabine in a panel of cancer cell lines. RASSF1A was identified by literature searches as being commonly silenced by DNA CpG methylation in 4 out of the 5 cell lines as indicated. Cells (A549, SKMES1, Du145 and 22Rv1) were treated with either decitabine or gemcitabine at the concentrations indicated, and expression of RASSF1A was examined by RT-PCR. Reactivation/upregulation of RASSF1A was observed to occur in 4 out of the 4 cell lines following decitabine treatment and in 3 out of the 4 cell lines following gemcitabine treatment.

cells treated with various concentrations of gemcitabine or decitabine. Gemcitabine was found to affect DNMT1 protein levels to different degrees for all the cell lines examined (Fig. 4E). 
A

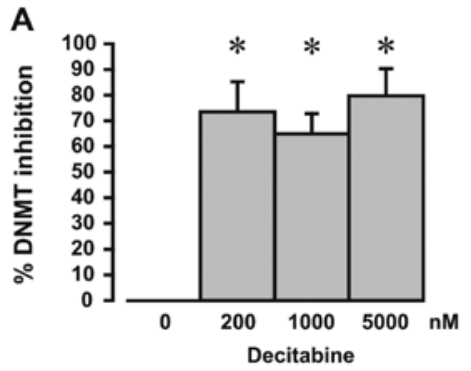

B

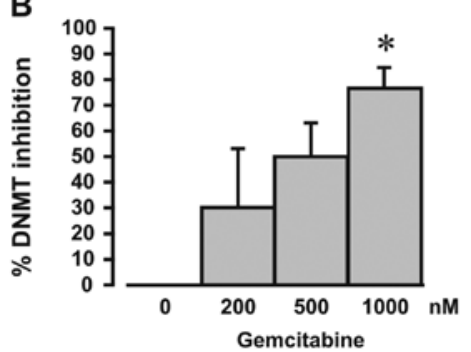

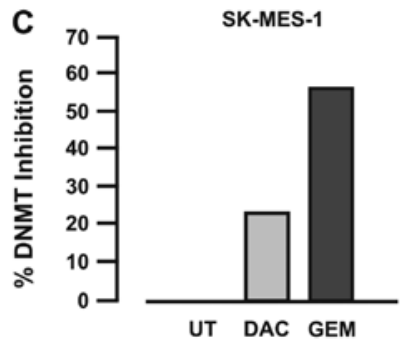

D $\quad$ H28

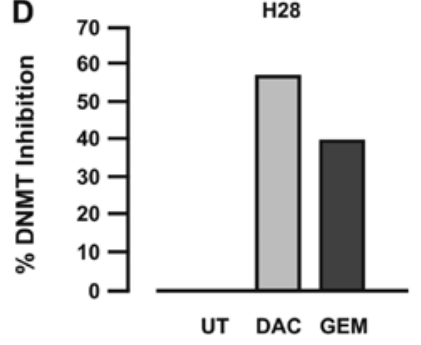

E

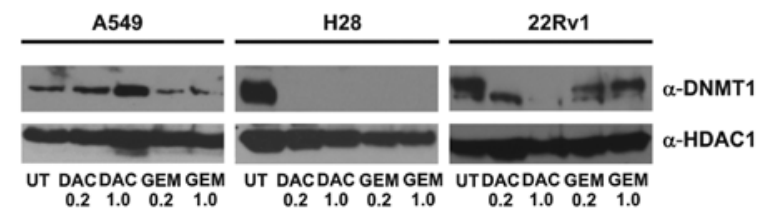

Figure 4. Gemcitabine directly inhibits DNA methyltransferase activity and affects DNMT1 protein levels. Both (A) decitabine and (B) gemcitabine inhibited DNMT activity in recombinant proteins incubated with either drug. DNA methyltransferase activity was measured using EpiQuik DNA methyltransferase activity/inhibition assay kit and \% inhibition was noted relative to untreated controls. Significance was determined using one-way analysis of variance (ANOVA). Following ANOVA a post hoc test was performed using the Dunnett's multiple comparisons test. Differences were considered significant at $\mathrm{P}<0.05$. Cells were treated with decitabine or gemcitabine, nuclear extracts were prepared and \% DNA methyltransferase inhibition was measured. Results are presented for (C) SK-MES-1 and (D) H28 cells (n=2 independent experiments). The results showed that overall DNA methyltransferase activity was inhibited by either drug. Similar results were obtained for A549 (data not shown). (E) Western blot analyses were performed on nuclear extracts of cells treated with decitabine or gemcitabine for DNMT1. HDAC1 was used as a loading control. Gemcitabine and decitabine were shown to affect the levels of DNMT1 to various degrees in the cell lines tested.

Gemcitabine does not alter global DNA CpG methylation or at the promoters of reactivated genes. Schafer and colleagues published data demonstrating that gemcitabine affects DNA methylation in cancer cells by inhibiting nucleotide excision repair (NER)-mediated DNA demethylation. Intriguingly, the authors showed that gemcitabine treatments did not affect global levels of DNA methylation but induced hypermethylation and silencing of MLH1 (34).

Using pyrosequencing of long interspersed nucleotide element (LINE-1) sequences, we examined global methylation in our cell lines. In agreement with Schafer et al (34), we did not observe any gross alterations in the global DNA CpG methylation following treatment with gemcitabine (Table III). For the lower concentration of decitabine tested $(0.2 \mu \mathrm{M})$ we noted a decrease in $\mathrm{CpG}$ methylation which did not occur in the cells treated at the higher concentration $(1 \mu \mathrm{M})$ in agreement with effects reported by others (35).

Next, we examined the effect of decitabine and gemcitabine on $\mathrm{CpG}$ methylation within RASSF1A. Again decitabine caused alterations to DNA CpG methylation levels at the lower concentration, yet there was no effect on methylation at the higher concentration (Table III). For the most part gemcitabine did not alter DNA CpG methylation for either concentration examined (Table III). Similar results were observed for VEGFR1 (data not shown).
GSTP1 has been previously shown to be downregulated by DNA CpG methylation (27-29). Given that we were able to demonstrate that both gemcitabine and decitabine reactivated the expression of GSTP1 in prostate cancer cell lines (Fig. 2), using MS-PCR, we examined whether or not there is a loss of methylation at the GSTP1 promoter in the 22Rv1 cell line. Our results showed a clear increase in the levels of unmethylated DNA at the GSTP1 promoter following treatment with either drug, indicating that there may be some changes to DNA CpG methylation occurring in cells treated with gemcitabine (Fig. 5).

\section{Discussion}

Gemcitabine is a well-established chemotherapy agent used as a single-agent treatment in pancreatic cancer, or in combination with various other agents in the treatment of many solid tumours. Its mode of action is generally considered to be that of an anti-metabolite acting to either inhibit ribonucleotide reductase (RNR) or by replacing cytidine during DNA replication.

Aberrant epigenetic regulation through DNA CpG methylation is a frequent event in cancer and reactivation of the target of methylation induced silencing 1 (TMS1) by DNA methyltransferase inhibitors (DNMTi) was found to enhance sensitivity to gemcitabine in pancreatic cancer cells (36). 
Table III. Percentage of methylation for LINE-1 and RASSF1A as determined by pyrosequencing.

\begin{tabular}{|c|c|c|c|}
\hline Cell line & Treatment & LINE-1 & RASSF1 \\
\hline \multirow[t]{5}{*}{ A549 } & UT & 55.8 & 85.2 \\
\hline & DAC 0.2 & 32.8 & 45.3 \\
\hline & DAC 1.0 & 51.3 & 79.2 \\
\hline & Gem 0.2 & 54.7 & 85.8 \\
\hline & Gem 1.0 & 55.7 & 86.2 \\
\hline \multirow[t]{4}{*}{ SKMES-1 } & UT & 53.1 & 7.3 \\
\hline & DAC 0.2 & 45.2 & 4.4 \\
\hline & DAC 1.0 & 52.7 & 6.5 \\
\hline & Gem 0.2 & 51.2 & 5.2 \\
\hline \multirow[t]{5}{*}{$\mathrm{H} 28$} & UT & 68.0 & 94.9 \\
\hline & DAC 0.2 & 47.8 & 65.8 \\
\hline & DAC 1.0 & 63.8 & 88.1 \\
\hline & Gem 0.2 & 69.6 & 94.6 \\
\hline & Gem 1.0 & 68.2 & 94.5 \\
\hline \multirow[t]{5}{*}{ Du145 } & UT & 65.2 & 71.2 \\
\hline & DAC 0.2 & 45.8 & 51.0 \\
\hline & DAC 1.0 & 56.5 & 64.0 \\
\hline & Gem 0.2 & 64.3 & 71.6 \\
\hline & Gem 1.0 & 66.0 & 71.9 \\
\hline \multirow[t]{5}{*}{$22 \mathrm{Rv} 1$} & UT & 50.0 & 83.8 \\
\hline & DAC 0.2 & 37.7 & 53.3 \\
\hline & DAC 1.0 & 42.5 & 61.2 \\
\hline & Gem 0.2 & 50.4 & 82.6 \\
\hline & Gem 1.0 & 52.0 & 74.3 \\
\hline
\end{tabular}

UT, untreated; DAC 0.2 , decitabine $0.2 \mu \mathrm{M}$; DAC 1.0 , decitabine $1.0 \mu \mathrm{M}$; Gem 0.2, gemcitabine $0.2 \mu \mathrm{M}$; Gem 1.0, gemcitabine $1.0 \mu \mathrm{M}$.

We noted that gemcitabine shares structural similarity with the FDA-approved DNMTi decitabine (Dacogen) (Fig. 1) and tested whether or not it has the ability to affect DNA CpG methylation. We showed that gemcitabine actively induces expression of epigenetically silenced genes in lung, mesothelioma (MPM) and prostate cancer cell lines (Figs. 2 and 3). We showed that gemcitabine directly inhibits DNA methyltransferases (Fig. 4). In addition, we also showed that gemcitabine depletes levels of DNMT1 similar to decitabine (Fig. 4). However, despite gemcitabine's ability to inhibit DNMTs, alter cellular DNMT1 protein levels and reactivate epigenetically silenced genes, there were few if any changes to DNA CpG methylation levels. We provide some evidence that active demethylation of an epigenetically silenced gene can occur (Fig. 5), but another mechanism may have to be invoked.

Schafer et al (34) published additional data demonstrating that gemcitabine affects DNA methylation in cancer cells by inhibiting nucleotide excision repair (NER)-mediated DNA demethylation. The authors showed that gemcitabine treatments did not affect global levels of DNA methylation

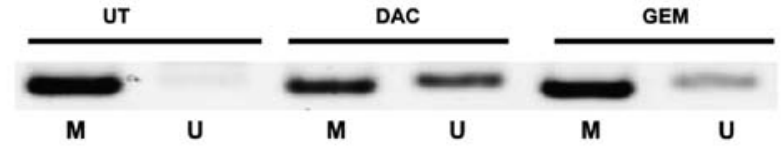

Figure 5. Gemcitabine affects DNA CpG methylation directly at the promoters of silenced genes. MS-PCR analysis of a known hypermethylated region of the GSTP1 promoter was carried out on bisulfite-modified DNA extracted from 22Rv1 cells treated as described. The results showed that in untreated cells the region was predominantly methylated (M). Following treatment there was a clear increase in the amount of unmethylated (U) PCR product in the cells treated with either drug. UT, untreated; DAC, decitabine; GEM, gemcitabine.

but induced hypermethylation and silencing of MLH1 (34). In agreement with the authors we also found that global levels of DNA CpG methylation were unaffected. In addition, methylation at the promoters of genes which could be reactivated following treatments with either decitabine or gemcitabine did not demonstrate any major changes in methylation status. This may in part be due to the concentrations of drugs used. For example, it has been established that high concentrations of decitabine have less effect on DNA methylation due to cytostatic effects, and our results using pyrosequencing confirm this demonstrating that changes in DNA methylation only occurred at the lower concentration of drug used (Table III). Using MTT assays we examined cellular proliferation across a range of concentrations (decitabine 200-5,000 nM; gemcitabine 50-1,000 $\mathrm{nM}$ ) in A549 and SKMES1 cells. While decitabine did not affect cellular proliferation to any appreciable effect at any concentration, gemcitabine caused significant decreases (of the order 25-50\%) in cellular proliferation (data not shown), which was not unexpected given the other known mechanisms of action of this drug.

Schafer et al (34) used a range of concentrations (34, 67 or $134 \mathrm{nM}$ ), whereas we used higher concentrations $(0.2-1 \mu \mathrm{M})$. It must be noted, however, that in gemcitabine infusion studies of patients with solid tumours, blood concentrations of gemcitabine at Day 1 of infusion varied between 18 and $77 \mu \mathrm{M}$ and at Day 15 between 13 and $90 \mu \mathrm{M}$ (37). As such the amount of gemcitabine used in our analysis was still below the levels utilised in patients.

The concentration of gemcitabine may become a critical determinant in how this chemotherapeutic agent is used in the clinic. For instance, in a study using MPM cells, gemcitabine inhibited the secretion of the proinflammatory cytokine IL-6 at drug concentrations that produced only small decreases in cell viability, whereas at higher doses a surge of IL-6 release was noted (38). We also observed an increase in the protumourigenic cytokine IL-23 in a NSCLC cell line treated with gemcitabine (Gray and O'Byrne, unpublished data).

How then does gemcitabine induce or reactivate epigenetically silenced genes. The nitrogen substitution of carbon 5 in the cytosine ring (Fig. 1) is involved in the mechanism of DNMT inactivation. As this nitrogen is not present in gemcitabine it may be that some sort of steric hindrance is involved affecting DNMTs and or methyl-binding proteins. Conceivably by inhibiting binding of methyl-binding protein complexes to DNA CpG sites this may relax chromatin and result in the re-expression of silenced genes. 
The results presented by us and Schafer et al (34) demonstrate a novel indication for gemcitabine and may have important implications in oncology as DNA methylation effects are potentially useful as biomarkers to either monitor response to therapy (39), or may have predictive value (40). In particular, the ability of gemcitabine to reactivate epigenetically silenced genes indicates a potential means to monitor and identify those patients who actually respond to gemcitabine treatment.

There are exciting new avenues which could be exploited, for example the use of low dose gemcitabine regimens combined with other epigenetic targeting drugs such histone deacetylase inhibitors. In addition low dose regimens may have the ability to sensitise resistant cancer to other cancer therapies. Further study will be required to fully delineate these possibilities.

\section{Acknowledgements}

Funding support for this project was provided by the Irish Cancer Society to Antoinette Perry.

\section{References}

1. Hidalgo M: Pancreatic cancer. N Engl J Med 362: 1605-1617, 2010

2. DeVita VT Jr: A step in the right direction. Nat Clin Pract Oncol 3: 641, 2006

3. Tkaczuk KH: Review of the contemporary cytotoxic and biologic combinations available for the treatment of metastatic breast cancer. Clin Ther 31: 2273-2289, 2009.

4. Goffin J, Lacchetti C, Ellis PM, Ung YC and Evans WK: First-line systemic chemotherapy in the treatment of advanced non-small cell lung cancer: a systematic review. J Thorac Oncol 5: 260-274, 2010.

5. Nagel S, Califano R, Thatcher N and Blackhall F: Gemcitabine and carboplatin in combination for the treatment of advanced metastatic, non-small cell lung cancer. Expert Opin Pharmacother 8: 3265-3275, 2007.

6. Fennell DA, Gaudino G, O'Byrne KJ, Mutti L and van Meerbeeck J: Advances in the systemic therapy of malignant pleural mesothelioma. Nat Clin Pract Oncol 5: 136-147, 2008.

7. Ray M and Kindler HL: Malignant pleural mesothelioma: an update on biomarkers and treatment. Chest 136: 888-896, 2009.

8. Jantscheff P, Esser N, Graeser R, et al: Liposomal gemcitabine (GemLip)-efficient drug against hormone-refractory Du145 and PC-3 prostate cancer xenografts. Prostate 69: 1151-1163, 2009.

9. Jantscheff P, Ziroli V, Esser N, et al: Anti-metastatic effects of liposomal gemcitabine in a human orthotopic $\mathrm{LNCaP}$ prostate cancer xenograft model. Clin Exp Metastasis 26: 981-992, 2009.

10. Plunkett W, Huang P, Xu YZ, Heinemann V, Grunewald R and Gandhi V: Gemcitabine: metabolism, mechanisms of action, and self-potentiation. Semin Oncol 22 (Suppl 11): 3-10, 1995.

11. Lawless MW, Norris S, O'Byrne KJ and Gray SG: Targeting histone deacetylases for the treatment of disease. J Cell Mol Med 13: 826-852, 2009.

12. O'Byrne KJ, Barr MP and Gray SG: The role of epigenetics in resistance to cisplatin chemotherapy in lung cancer. Cancers 3 : 1426-1453, 2011.

13. Cai D, Shames DS, Raso MG, et al: Steroid receptor coactivator-3 expression in lung cancer and its role in the regulation of cancer cell survival and proliferation. Cancer Res 70: 6477-6485, 2010.

14. Hajji N, Wallenborg K, Vlachos P, Fullgrabe J, Hermanson O and Joseph B: Opposing effects of hMOF and SIRT1 on H4K16 acetylation and the sensitivity to the topoisomerase II inhibitor etoposide. Oncogene 29: 2192-2204, 2010.

15. Zhao W, Zhang Q, Kang X, Jin S and Lou C: AIB1 is required for the acquisition of epithelial growth factor receptor-mediated tamoxifen resistance in breast cancer cells. Biochem Biophys Res Commun 380: 699-704, 2009.

16. Baylin SB and Jones PA: A decade of exploring the cancer epigenome - biological and translational implications. Nat Rev Cancer 11: 726-734, 2011.
17. Gore SD, Jones $\mathrm{C}$ and Kirkpatrick P: Decitabine. Nat Rev Drug Discov 5: 891-892, 2006.

18. Kaminskas E, Farrell A, Abraham S, et al: Approval summary: azacitidine for treatment of myelodysplastic syndrome subtypes. Clin Cancer Res 11: 3604-3608, 2005.

19. Yokochi T and Robertson KD: Doxorubicin inhibits DNMT1, resulting in conditional apoptosis. Mol Pharmacol 66: 1415-1420, 2004.

20. Gray SG, Al-Sarraf N, Baird AM, Cathcart MC, McGovern E and O'Byrne KJ: Regulation of EP receptors in non-small cell lung cancer by epigenetic modifications. Eur J Cancer 45: 3087-3097, 2009.

21. Laird PW, Zijderveld A, Linders K, Rudnicki MA, Jaenisch R and Berns A: Simplified mammalian DNA isolation procedure. Nucleic Acids Res 19: 4293, 1991.

22. Daskalos A, Logotheti S, Markopoulou S, et al: Global DNA hypomethylation-induced DeltaNp73 transcriptional activation in non-small cell lung cancer. Cancer Lett 300: 79-86, 2011.

23. Daskalos A, Nikolaidis G, Xinarianos G, et al: Hypomethylation of retrotransposable elements correlates with genomic instability in non-small cell lung cancer. Int J Cancer 124: 81-87, 2009.

24. Daskalos A, Oleksiewicz U, Filia A, et al: UHRF1-mediated tumor suppressor gene inactivation in nonsmall cell lung cancer. Cancer 117: 1027-1037, 2011

25. He B, Lee AY, Dadfarmay S, et al: Secreted frizzled-related protein 4 is silenced by hypermethylation and induces apoptosis in beta-catenin-deficient human mesothelioma cells. Cancer Res 65: 743-748, 2005.

26. Lee AY, He B, You L, et al: Expression of the secreted frizzled-related protein gene family is downregulated in human mesothelioma. Oncogene 23: 6672-6676, 2004.

27. Nelson WG, De Marzo AM and Yegnasubramanian S: Epigenetic alterations in human prostate cancers. Endocrinology 150: 3991-4002, 2009.

28. Perry AS, Liyanage H, Lawler M and Woodson K: Discovery of DNA hypermethylation using a DHPLC screening strategy. Epigenetics 2: 43-49, 2007.

29. Perry AS, Loftus B, Moroose R, et al: In silico mining identifies IGFBP3 as a novel target of methylation in prostate cancer. Br J Cancer 96: 1587-1594, 2007.

30. Endoh H, Yatabe Y, Shimizu S, et al: RASSF1A gene inactivation in non-small cell lung cancer and its clinical implication. Int J Cancer 106: 45-51, 2003.

31. Kuzmin I, Gillespie JW, Protopopov A, et al: The RASSF1A tumor suppressor gene is inactivated in prostate tumors and suppresses growth of prostate carcinoma cells. Cancer Res 62: 3498-3502, 2002.

32. Toyooka S, Pass HI, Shivapurkar N, et al: Aberrant methylation and simian virus 40 tag sequences in malignant mesothelioma. Cancer Res 61: 5727-5730, 2001.

33. Ghoshal K, Datta J, Majumder S, et al: 5-Aza-deoxycytidine induces selective degradation of DNA methyltransferase 1 by a proteasomal pathway that requires the KEN box, bromoadjacent homology domain, and nuclear localization signal. Mol Cell Biol 25: 4727-4741, 2005.

34. Schafer A, Schomacher L, Barreto G, Doderlein G and Niehrs C: Gemcitabine functions epigenetically by inhibiting repair mediated DNA demethylation. PLoS One 5: e14060, 2010.

35. Tsai HC, Li H, Van Neste L, et al: Transient low doses of DNA-demethylating agents exert durable antitumor effects on hematological and epithelial tumor cells. Cancer Cell 21: 430-446, 2012.

36. Ramachandran K, Miller H, Gordian E, Rocha-Lima C and Singal R: Methylation-mediated silencing of TMS1 in pancreatic cancer and its potential contribution to chemosensitivity. Anticancer Res 30: 3919-3925, 2010.

37. de Lange SM, van der Born K, Kroep JR, et al: No evidence of gemcitabine accumulation during weekly administration. Eur J Clin Pharmacol 61: 843-849, 2005.

38. McLaren BR, Robinson BW and Lake RA: New chemotherapeutics in malignant mesothelioma: effects on cell growth and IL-6 production. Cancer Chemother Pharmacol 45: 502-508, 2000.

39. Levenson VV: DNA methylation as a universal biomarker. Expert Rev Mol Diagn 10: 481-488, 2010.

40. Salazar F, Molina MA, Sanchez-Ronco M, et al: First-line therapy and methylation status of CHFR in serum influence outcome to chemotherapy versus EGFR tyrosine kinase inhibitors as second-line therapy in stage IV non-small-cell lung cancer patients. Lung Cancer 72: 84-91, 2011. 